\title{
Nuevos datos morfométricos para el Lago de Sanabria
}

\author{
J. C. Vega ${ }^{1}$, C. De $\operatorname{Hoyos}^{2}$, J. J. Aldasoro³ ${ }^{3}$ J. De Miguel ${ }^{4}$ y H. Fraile 5 \\ ${ }^{1}$ Parque Natural del Lago de Sanabria. Consejería de Medio Ambiente. Junta de Castilla y León. \\ jcvega@ctv.es \\ ${ }^{2}$ Centro de Estudios Hidrográficos. CEDEX. Paseo Bajo de la Virgen del Puerto, 3. 28005 Madrid. cari- \\ dad.dehoyos@cedex.es \\ ${ }^{3}$ Real Jardín Botánico. CSIC. Plaza de Murillo 2. 28014 Madrid. aldasoro@ma-rjb.csic.es \\ ${ }^{4} \mathrm{C} /$ Victor de la Serna, 22-8 D. 28016 Madrid. \\ ${ }^{5}$ C/ Zabalbide $15,3^{\circ}$ Izq. 48006 Bilbao
}

\section{RESUMEN}

Se presenta un nuevo mapa batimétrico del lago de Sanabria, con curvas de nivel situadas a cada metro de profundidad y algunos parametros morfométricos. La forma de este lago, basándonos en la curva hipsográfica relativa del área frente a la profundidad es cóncava $(\mathrm{C})$. Este lago tiene altos valores de los parámetros: desarrollo del volumen $\left(\mathrm{D}_{\mathrm{v}}\right)$ y la relación de profundidad (cociente profundidad media / profundidad máxima: $\mathrm{z}_{\mathrm{m}} / \mathrm{z}_{\mathrm{max}}$ ), esto refleja su morfometría con un fondo considerablemente plano y grandes pendientes desde las orillas hacia el mismo, como ocurre en algunos tipos de lagos de origen glaciar. La profundidad relativa $\left(\mathrm{Z}_{\mathrm{r}}\right)$ es intermedia y los índices: área de la cuenca / volumen del lago $\left(\mathrm{A}_{\mathrm{c}} / \mathrm{V}\right)$ y área de la cuenca / área del lago $\left(\mathrm{A}_{\mathrm{c}} / \mathrm{A}\right)$ son bastante altos, si los comparamos con otros lagos. El valor de estos parámetros indica la gran influencia de los factores externos sobre la dinámica del lago.

Palabras clave: lagos de origen glaciar, mapa batimétrico, morfometría

\begin{abstract}
A new bathymetric map of Lake Sanabria is presented, showing depth isolines each meter and some morphometric parameters. The lake form, based on the relative hypsographic curve (cumulative area-depth), is concave (C). This lake shows high values of volume development $\left(D_{v}\right)$ and of the depth relation (mean depth / maximal depth ratio: $z_{m} / z_{\text {max }}$ ), which indicates a nearly flat bottom and high slopes in the shores, like those of some types of glacial lakes. The relative depth $\left(Z_{r}\right)$ has an intermediate value and the indexes: basin area / lake volume $\left(A_{c} / V\right)$ and basin area / lake area $\left(A_{c} / A\right)$ are quite high, when compared to other lakes. The values of these parameters indicate the large influence of external factors on the lake dynamics.
\end{abstract}

Keywords: glacial lakes, bathymetric map, morphometry

\section{INTRODUCCIÓN}

El lago de Sanabria está situado en el NW de la Península Ibérica $\left(42^{\circ} 07^{\prime} 30^{\prime \prime} \mathrm{N}, 06^{\circ} 43^{\prime} 00^{\prime \prime} \mathrm{W}\right)$ a $1000 \mathrm{~m}$ sobre el nivel del mar. Ocupa una depresión glaciar en el valle del río Tera; este río pertenece a la cuenca del río Duero y nace en la confluencia de las Sierras Segundera y Cabrera. Los primeros datos sobre la batimetría del lago de Sanabria fueron recogidos por Taboada (1913); este autor realizó el primer mapa batimétrico del lago basado en tres transectos (unos 50 sondeos). Fue también Taboada quién prime- ro definió el origen glaciar del lago e hizo una amplia descripción del sistema de morrenas que lo cierran. En la década de los años 50 se hicieron otros levantamientos batimétricos relacionados con proyectos de aprovechamiento hidroeléctrico. Posteriormente, a partir de 90 sondeos, se realizó una nueva batimetría más detallada (Aldasoro et al., 1992; Vega et al., 1992) con curvas de nivel cada $5 \mathrm{~m}$ y una descripción morfométrica del lago basada en la misma (De Hoyos, 1996). El presente trabajo amplía los anteriores con un nuevo mapa batimétrico basado en 48 transectos continuos, que permiten 
definir curvas de nivel cada metro de profundidad, y con nuevos parámetros morfométricos.

\section{METODOLOGÍA}

Se han realizado 48 transectos con una sonda Furuno-2000. Después de normalizados, estos perfiles batimétricos se introdujeron digitalizados en el programa AutoCAD-14 sobre un mapa del lago de Sanabria extraído a partir de una fotografía aérea del vuelo del Ministerio de Hacienda de 1980. La escala original de este vuelo es de 1:18.000. A partir de la fotografía aérea se realizó una ampliación cuya escala se obtuvo realizando mediciones sobre el terreno de varios tramos dispuestos en ángulo recto de las carreteras que aparecen en la imagen. La escala, así obtenida, fue de 1:9524, normalizada posteriormente a 1:10 000 con el Auto-CAD. A partir de las curvas batimétricas se trazaron las isolíneas de las cotas metro a metro desde 0 hasta $51 \mathrm{~m}$ de profundidad, obteniéndose a continuación superficies, perímetros y volúmenes de cada corte.

\section{RESULTADOS}

\section{Geomorfología y origen del lago}

La cuenca que drena hacia el lago tiene una superficie de $127.3 \mathrm{~km}^{2}$ (De Hoyos, 1996) y está formada por dos zonas, una alargada en dirección norte-sur, siguiendo la cuenca del río Tera y otra en dirección oeste-este, cuyas aguas llegan a los ríos Segundera y Cárdena, ríos que desembocan en el río Tera antes de su entrada en el lago. La parte alta de la cuenca está formada por una meseta situada en la zona superior de las sierras Segundera y Cabrera, desde esta zona hasta el lago el terreno tiene una pronunciada inclinación (Fig. 1). En el norte la pendiente llega hasta la orilla del lago (Figs. 1A y 1B). La orilla oeste tiene una pendiente menos pronunciada, existe una zona llana desde la orilla del lago hasta la fuerte inclinación que conecta con la meseta de la sierra (Fig. 1C), esta zona está rellena de sedimentos aportados por el río. Al este el lago se cierra con morrenas glaciares (Fig. 1C).

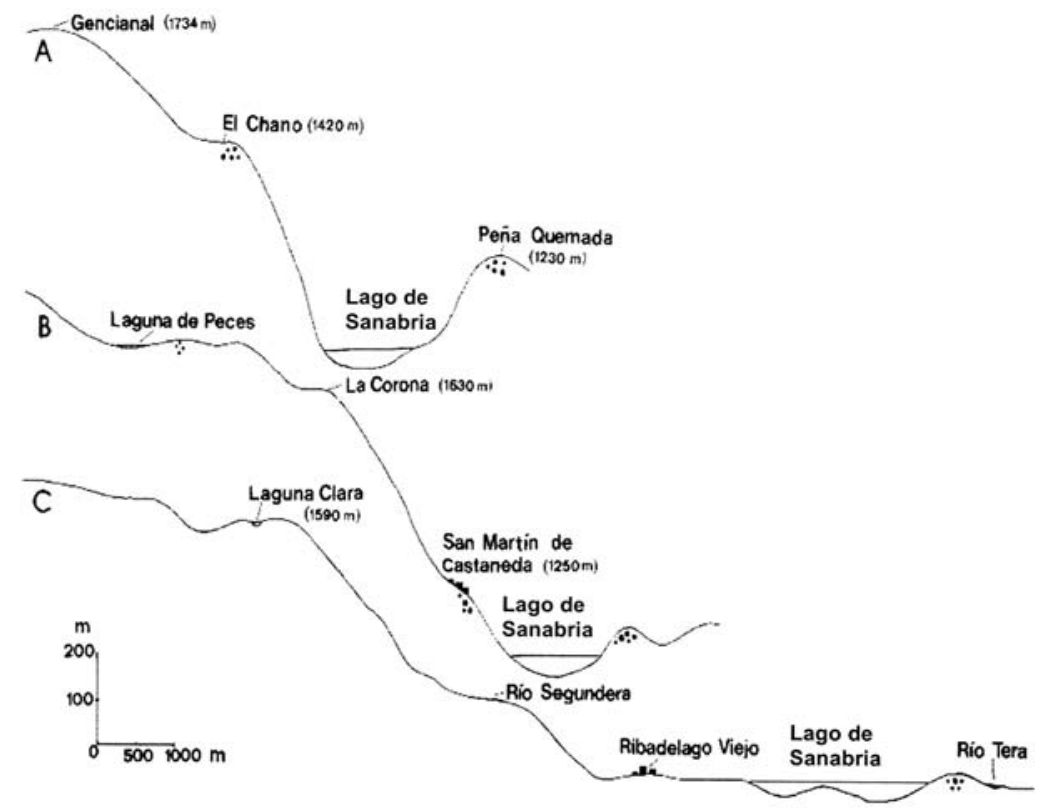

Figura 1. Cortes topográficos de la cuenca del lago de Sanabria. A y B son cortes N-S que atraviesan el lago transversalmente por las cubetas W y E respectivamente. C es un corte W-E que atraviesa el lago longitudinalmente. Topographic cross-sections of Lake Sanabria basin. A and B are Nord-South cross-sections which cross the basin transversally. C is a cross-section which crosses the lake longitudinally. 
La cubeta que ocupa el lago de Sanabria se ha formado a partir de un valle fluvial preglaciar formado por el río Tera y modificado más tarde por acción glaciar. Durante la glaciación del Würm se acumularon en las sierras Segundera y Cabrera grandes cantidades de hielo originando un glaciar de meseta o casquete situado a $1500-1700 \mathrm{~m}$ de altitud. Este glaciar de meseta alimentaba varias lenguas de hielo que bajaban aprovechando anteriores valles fluviales. La confluencia de la lengua glaciar del Tera con la que descendía por los valles del Segundera y del Cárdena, desde los $1700 \mathrm{~m}$ del altiplano hasta la altura de Ribadelago, dio lugar a una acumulación de hielo de cerca de 300 $\mathrm{m}$ de espesor en algunos puntos. Esta lengua glaciar arrancó y arrastró grandes cantidades de materiales que originaron las morrenas laterales que se observan actualmente a ambos lados del valle del lago (Figs. 1A y 1B) y la serie de morrenas frontales (siete) que cierran el valle y marcan los sucesivos retrocesos del glaciar. La mas alejada se encuentra en las proximidades del pueblo de Pedrazales (Vega \& Aldasoro, 1994), mientras que el más próximo de estos arcos morrénicos es el que cierra la cubeta del lago.

Según la clasificación que Hutchinson (1957) hace de los lagos según su origen, el lago de Sanabria pertenece al grupo 28c (De Hoyos, 1996), son lagos formados por la actividad de glaciares que descendieron desde las montañas hasta un nivel inferior; es el único lago de este tipo (llamados lagos marginales o de pié de monte) que existe en la Península Ibérica (Margalef, 1983).

\section{Morfometría}

La morfometría de un lago está relacionada principalmente con su origen, historia y con las características geológicas de su cubeta y de su cuenca. Estos condicionantes influyen a su vez en el comportamiento térmico, régimen hidrológico y dinámica del mismo. La morfología actual del Lago de Sanabria es la consecuencia de la interacción entre las fuerzas del hielo del glaciar que hemos comentado anteriormente, la escasa pendiente del valle en este tramo y la litología de la cubeta en una zona que es de transición entre granitos y gneises. A estas fuerzas originales se añaden posteriormente los derrumbes de las laderas, la erosión fluvial de la cuenca directa, la acción del oleaje en las orillas mas expuestas al viento del oeste y los aportes fluviales del Tera que han rellenado paulatinamente la orilla oeste del lago.

En la figura 2 se puede ver el nuevo mapa batimétrico. Longitudinalmente el lago se divide en dos cubetas. La cubeta este es la más profunda, su profundidad máxima $\left(Z_{\max }\right)$ es de $51 \mathrm{~m}$. La $Z_{\max }$ de la cubeta este es de $46 \mathrm{~m}$. Estas dos máximas profundidades se corresponden posiblemente con los dos centros de máxima presión de los hielos. La presencia de más de una zona profunda, con barreras separando las cubetas, se produce también en otros lagos formados en un valle sobre excavado por la acción de un glaciar (Hutchinson, 1957). Esta estructura puede explicarse por cambios en la pendiente del valle y por la existencia de materiales con diferente grado de resistencia a lo largo del recorrido del hielo.

Las secciones transversales del mapa batimétrico que se señalan en la figura 2 se pueden ver en la figura 3 y muestran un valle típico de erosión glaciar, en forma de U; las orillas norte y sur son casi verticales. La máxima pendiente se encuentra en la orilla sur de la cubeta este donde la ladera desciende hasta los $45 \mathrm{~m}$ de profundidad. La sección longitudinal (perfil A) muestra que tanto la orilla este como la oeste tiene una inclinación más suave. En la orilla oeste esta variación gradual de la profundidad se debe a los sedimentos que el río Tera va depositando y que ya rellenaron el lago por su parte occidental, en la zona de Ribadelago nuevo (Fig. 1), formando tierras de aluvión que actualmente se cultivan. Parecen existir en esta orilla, cuatro anteriores entradas de agua al lago, la primera inmediatamente al norte del peñón del Castro, en Seoane, y las otras tres al sur de este promontorio entre el peñón y la actual desembocadura del río Tera. En la orilla este, el oleaje predominante, que es el que originan los vientos del oeste-noroeste, ha erosionado la morrena de cierre y los materiales finos originados se han depositado a lo largo de una plataforma situada aproximadamente a $5 \mathrm{~m}$ de profundidad y paralela a toda la orilla. 


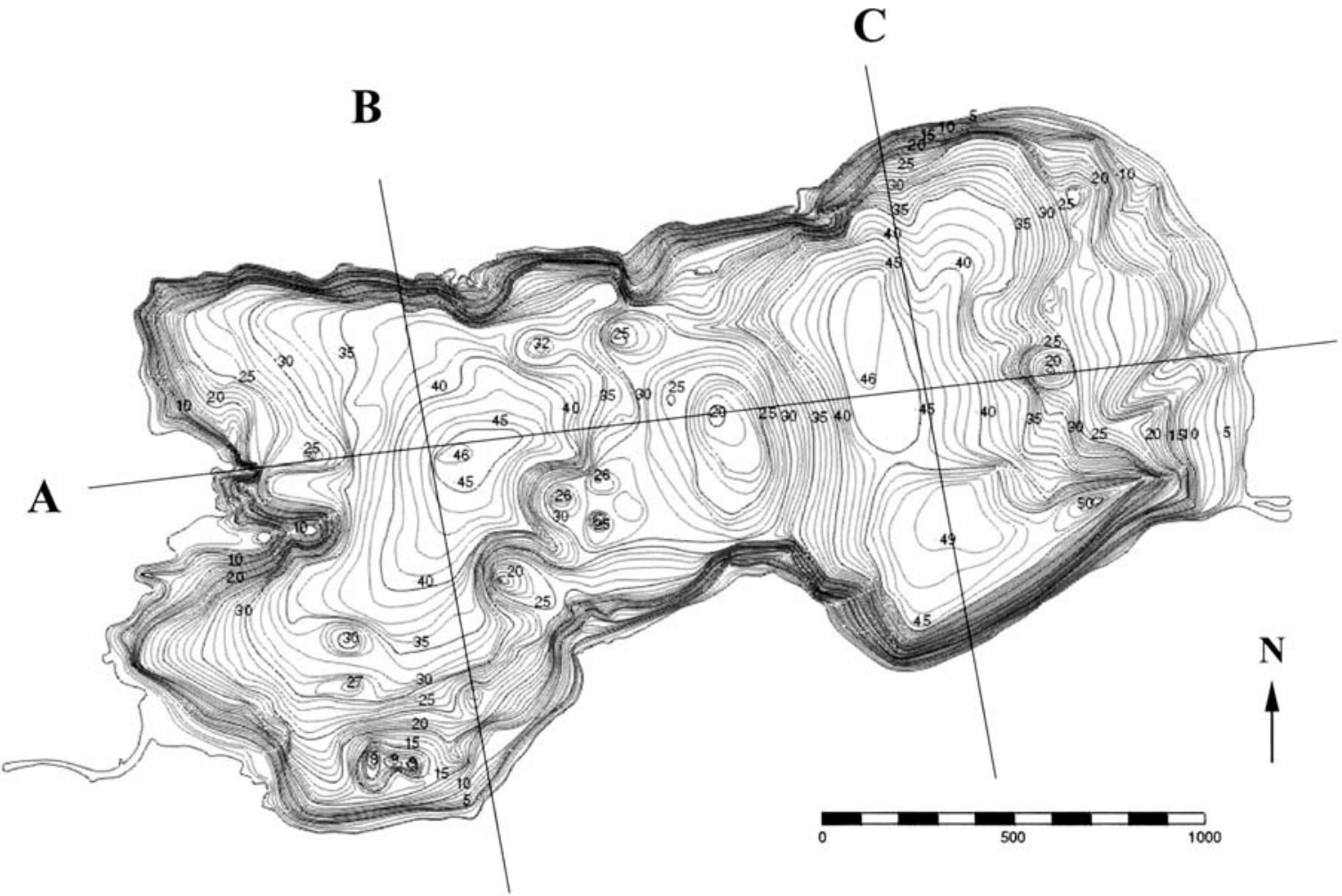

Figura 2. Mapa batimétrico del lago de Sanabria. Las líneas A, B y C corresponden a los perfiles A, B y C de la figura 3. Bathymetric map of Lake Sanabria. The lines A, B and C correspond to the sections $A, B$ and $C$ shown in figure 3.
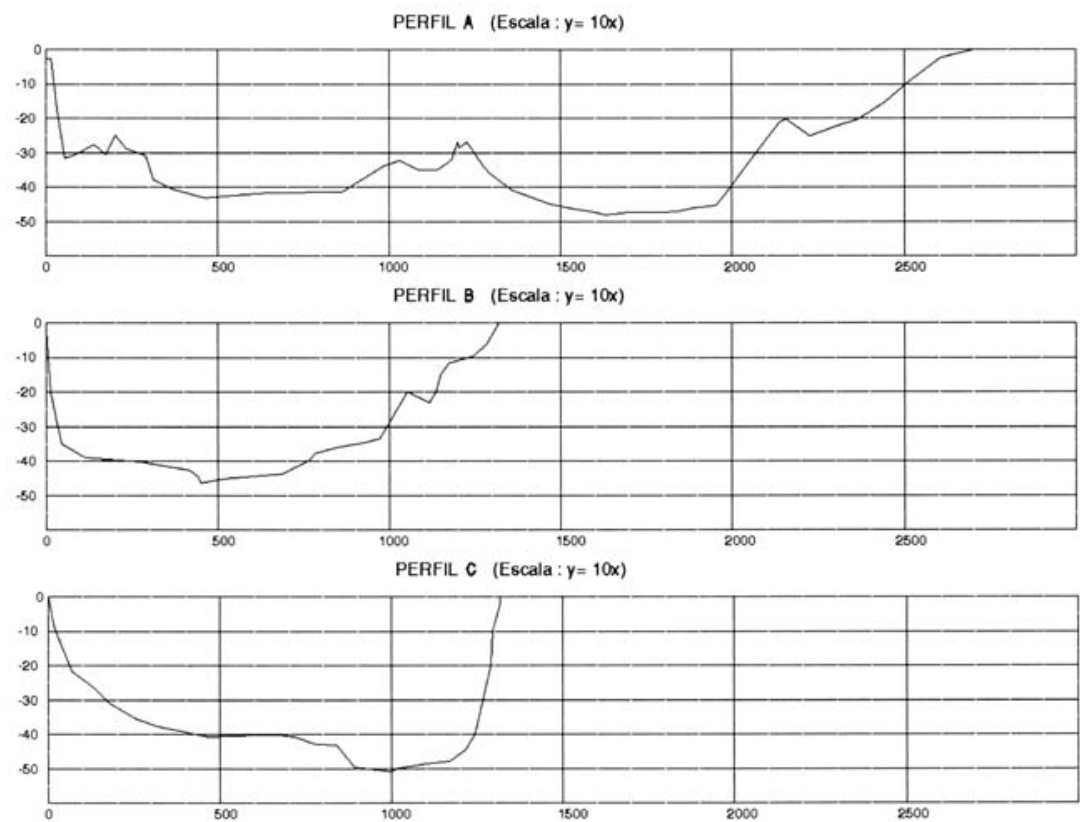

Figura 3. Sección longitudinal y transversal (Fig. 2) del lago de Sanabria. Longitudinal and cross-sections (Fig. 2) of Lake Sanabria. 
En la cubeta oeste y muy próxima a la costa, se encuentra una pequeña isla $\left(700 \mathrm{~m}^{2}\right)$ formada por materiales graníticos, prolongación del peñón del Castro, sobre la que se han acumulado de forma artificial cierta cantidad de rocas, y existen restos de un antiguo embarcadero. En la nueva batimetría se descubre hacia el este del peñasco y sumergida a unos $10 \mathrm{~m}$ de profundidad una réplica casi exacta de esta isla. Desde aquí el fondo cae bruscamente hasta $35 \mathrm{~m}$ de profundidad. Tanto en la cubeta este cómo en la oeste, la conformación de los fondos por debajo de $\operatorname{los} 35 \mathrm{~m}$ de profundidad, con pendientes mas suaves, responde sobre todo a la acumulación de sedimentos orgánicos, lodos, que con una potencia estimada de $12 \mathrm{~m}$, van rellenando la cubeta del lago a una tasa aproximada de $1 \mathrm{~mm} /$ año(de Hoyo, comunicación personal).

La cubeta oeste muestra una mayor abundancia de promontorios rocosos, elevados unos $20 \mathrm{~m}$ sobre los fondos inmediatos. Llama la atención uno de ellos, que situado en las proximidades de la orilla sur de esta cubeta se alza, dividido en tres peñascos, hasta apenas $8 \mathrm{~m}$ de la superficie. La unión de las dos cubetas se realiza por encima de la isolínea de $-30 \mathrm{~m}$, al norte y al sur del promontorio de grandes dimensiones que separa ambas cubetas con una altura que alcanza en el centro unos $10 \mathrm{~m}$ sobre los fondos inmediatos.

La cubeta este alberga la máxima profundidad del lago, $51 \mathrm{~m}$, en el emplazamiento descubierto por Taboada en 1913. En la orilla este de esta cubeta y correspondiéndose con las escotaduras que presenta en superficie la morrena frontal se observan, en las profundidades inmediatas, restos de canales posiblemente formados por erosión a contracorriente de dos antiguos cauces de salida, uno en la esquina norte (La Folgosa) y otro en la mitad de la orilla este (Los Arenales). Este mismo fenómeno de erosión se observa en la actual salida del Tera, en la esquina sur de esta orilla.

En la tabla 1 se muestran algunas características e índices morfométricos del lago de Sanabria que han sido estimados de acuerdo con Hutchinson (1957) y Hakanson (1981). A partir del mapa batimétrico hemos calculado las
Tabla 1. Parámetros morfométricos del lago de Sanabria. Morphometric parameters of Sanabria lake.

\begin{tabular}{|c|c|}
\hline \multicolumn{2}{|l|}{ PARÁMETROS MORFOMÉTRICOS } \\
\hline Área del lago (A) & $3475798 \mathrm{~m}^{2}$ \\
\hline Área de la cuenca $\left(\mathrm{A}_{\mathrm{c}}\right)$ & $12730 \mathrm{hm}^{2}$ \\
\hline $\begin{array}{l}\text { Porcentaje de área de la cuenca } \\
\text { ocupada por el lago (A/Ac) }\end{array}$ & $2.73 \%$ \\
\hline Volumen (V) & $96289887 \mathrm{~m}^{3}$ \\
\hline Longitud máxima $\left(\mathrm{L}_{\max }\right)$ & $3160 \mathrm{~m}$ \\
\hline Anchura máxima (cubeta $E)\left(B_{\max }\right)$ & $1530 \mathrm{~m}$ \\
\hline Anchura máxima (cubeta W) & $1500 \mathrm{~m}$ \\
\hline Anchura mínima & $900 \mathrm{~m}$ \\
\hline Anchura media $\left(\mathrm{B}_{\mathrm{m}}\right)$ & $1099.9 \mathrm{~m}$ \\
\hline Profundidad máxima (cubeta $E)\left(Z_{\max }\right)$ & $51 \mathrm{~m}$ \\
\hline Profundidad máxima (cubeta W) & $46 \mathrm{~m}$ \\
\hline Profundidad media $\left(Z_{\mathrm{m}}\right)$ & $27,7 \mathrm{~m}$ \\
\hline Profundidad relativa $\left(Z_{\mathrm{r}}\right)$ & $2,42 \%$ \\
\hline Profundidad mediana $\left(\mathrm{Z}_{50}\right)$ & $31 \mathrm{~m}$ \\
\hline Longitud de la línea de costa (L) & $9518 \mathrm{~m}$ \\
\hline Desarrollo de la línea de costa $\left(\mathrm{D}_{\mathrm{L}}\right)$ & 1.44 \\
\hline Desarrollo del volumen $\left(\mathrm{D}_{\mathrm{v}}\right)$ & 1.63 \\
\hline $\mathrm{z}_{\mathrm{m}} / \mathrm{z}_{\max }$ & 0.54 \\
\hline $\mathrm{A}_{\mathrm{c}} / \mathrm{A}$ & 36.62 \\
\hline $\mathrm{A}_{\mathrm{c}} / \mathrm{V}$ & $1.32 \mathrm{~m}^{-1}$ \\
\hline
\end{tabular}
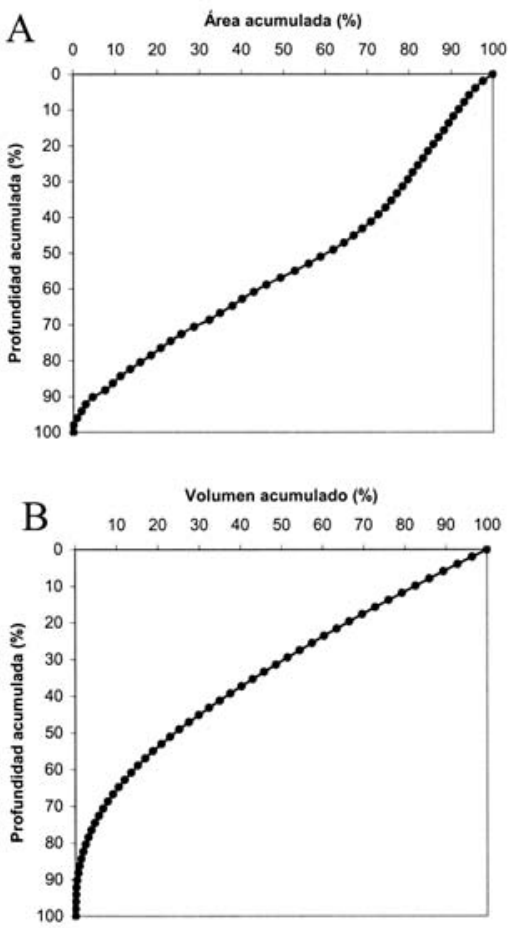

Figura 4. A.- Curva hipsográfica relativa. B.- Curva de volumenes relativos del lago de Sanabria. A.- Relative hipsographic curve. B.- Relative volumes curve for Lake Sanabria. 


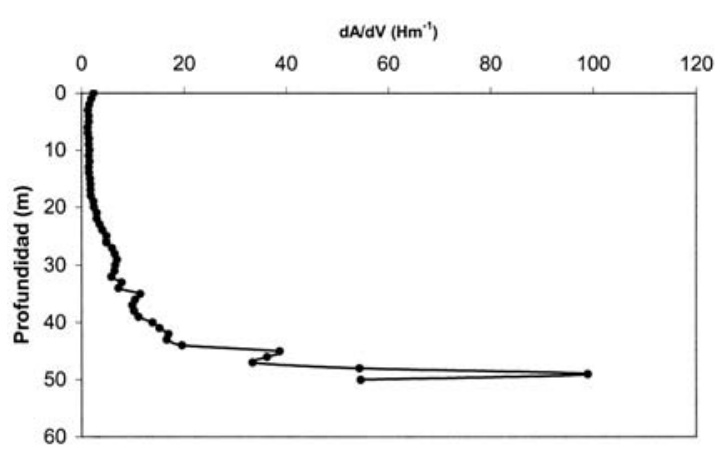

Figura 5. Relación del incremento del área / incremento del volumen respecto de la profundidad. Plot showing the increase of area / increase of volume vs. depth.

superficies que corresponden a cada uno de los $51 \mathrm{~m}$ de profundidad y los volúmenes de las masas de agua comprendidas desde cada una de esas superficies hasta el fondo del lago. La variación del área y del volumen, con relación a la profundidad, se puede ver en las figuras 4 y 5 . En la figura 5 se observan las interacciones que existen entre el incremento del área dividido entre el incremento del volumen y la profundidad. Estas figuras indican que a partir de los $25 \mathrm{~m}$ aumenta la importancia de la interacción entre el agua y el sedimento.
La forma del lago de Sanabria, atendiendo a la clasificación propuesta por Hakanson (1981) en función de la curva hipsográfica relativa del área versus profundidad (Fig. 4) es cóncava (C). En lagos con curvas hipsográficas relativas cóncavas la profundidad media es menor que la profundidad mediana (Tabla 1).

La relación de profundidad (cociente entre la profundidad media y la profundidad máxima: $\left.\mathrm{z}_{\mathrm{m}} / \mathrm{z}_{\mathrm{max}}\right)$ es un buen descriptor de la forma de la cubeta y en el caso del lago de Sanabria se podría concluir que se aproxima a una forma parabólica $\left(\mathrm{z}_{\mathrm{m}} / \mathrm{z}_{\max }=0.5\right)$. El alto valor relativo de $z_{m} / z_{\text {max }} y$ del desarrollo del volumen $\left(D_{v}\right)$ se corresponden con la morfometría del lago: un fondo casi plano y grandes pendientes desde las orillas hacia el mismo. Altos valores de ambos parámetros se dan, además de en algunos lagos de origen glaciar, en los que ocupan la caldera de un volcán, como el lago Crater (Tabla 2).

El desarrollo de la línea de costa $\left(\mathrm{D}_{\mathrm{L}}\right)$ está relacionado con el efecto de los procesos litorales. Este parámetro en el lago de Sanabria no es muy alto, si lo comparamos con el de otros lagos. El índice área de la cuenca / volumen $\left(\mathrm{A}_{\mathrm{c}} / \mathrm{V}\right)$ se puede relacionar con los parámetros tróficos (Catalán, 1987); el aporte externo de nutrientes

Tabla 2. Parámetros morfométricos de algunos lagos. Morphometric parameters of some lakes.

\begin{tabular}{|c|c|c|c|c|c|c|c|c|}
\hline & $\begin{array}{c}\text { Area del lago, } \\
\mathbf{A} \\
\left(\mathrm{km}^{2}\right)\end{array}$ & $\begin{array}{c}\text { Volumen, } \\
\text { V } \\
\left(\mathbf{k m}^{\mathbf{3}}\right)\end{array}$ & $\begin{array}{l}\text { Profundidad } \\
\text { máxima, } \\
\mathrm{Z}_{\max } \\
\text { (m) }\end{array}$ & 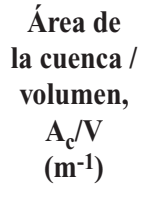 & $\begin{array}{c}\text { Área de la } \\
\text { cuenca / } \\
\text { area del } \\
\text { lago, } \\
\mathbf{A}_{\mathrm{c}} / \mathrm{A}\end{array}$ & $\begin{array}{c}\text { Profundidad } \\
\text { relativa, } \\
\mathrm{Z}_{\mathrm{r}} \\
(\%)\end{array}$ & $\begin{array}{c}\text { Desarrollo } \\
\text { del volumen, } \\
\mathbf{D}_{\mathrm{v}}\end{array}$ & Referencia \\
\hline L. Sanabria & 3.46 & 0.09 & 51 & 1.36 & 36.78 & 2.4 & 1.63 & este trabajo \\
\hline L. Redó & 0.24 & 0.007 & 73 & 0.2 & 6.5 & 13 & 1.3 & Catalán, 1987 \\
\hline L. Bañolas & 1.06 & 0.017 & 40 & - & - & 3.4 & 1.18 & Planas, 1973 \\
\hline L. Arreo & 0.065 & 0.00035 & 24.8 & 14.04 & 74.8 & 8.57 & 0.64 & Rico et al. , 1995 \\
\hline L. Como & 145.9 & 22.5 & 410 & 0.2 & 30.9 & 3.01 & 1.1 & Ambrosetti et al., 1992 \\
\hline L. Maggiore & 212.5 & 37.5 & 370 & 0.18 & 31.05 & 2.25 & 1.4 & Ambrosetti et al., 1992 \\
\hline L. Garda & 367.9 & 49.03 & 350 & 0.05 & 6.14 & 1.62 & 1.1 & Ambrosetti et al., 1992 \\
\hline L. Lugano S & 27.5 & 4.69 & 288 & 0.06 & 10.81 & 4.87 & 1.7 & Ambrosetti et al., 1992 \\
\hline L. Iseo & 61.8 & 7.6 & 251 & 0.23 & 28.09 & 2.83 & 1.4 & Ambrosetti et al., 1992 \\
\hline L. Baikal & 31500 & 23000 & 1741 & - & - & 0.85 & 1.2 & Hutchinson, 1957 \\
\hline L. Crater & 55 & 20 & 608 & - & - & 7.29 & 1.79 & Hutchinson, 1957 \\
\hline L. Tahoe & 499 & 124 & 501 & - & - & 1.99 & 1.49 & Hutchinson, 1957 \\
\hline L. Great Slave & 30000 & - & 614 & - & - & 0.31 & - & Hutchinson, 1957 \\
\hline
\end{tabular}


desde la superficie de la cuenca hacía el lago está influido por este índice, de manera que a un valor más pequeño del índice, más oligotrofia. En el lago de Sanabria la relación entre la cuenca de drenaje y el volumen del lago $\left(\mathrm{A}_{\mathrm{c}} /\right.$ $\mathrm{V})$ o su área $\left(\mathrm{A}_{\mathrm{c}} / \mathrm{A}\right)$ son bastante altos (Tablas 1 y 2), sin embargo es un lago oligotrófico. Esto es consecuencia del substrato poco soluble y de la escasa intervención humana en la cuenca (De Hoyos, 1996). Estos parámetros indican la gran influencia que tendrían diversos tipos de intervenciones o modificaciones de la cuenca sobre el estado trófico del lago.

La relación entre el área del lago, por donde entra la energía desde el exterior, y la profundidad, a través de la cual se disipa la energía, tiene una importancia fundamental en la dinámica biológica del lago (Catalán, 1987); esta relación se puede expresar numéricamente con la profundidad relativa $\left(Z_{r}\right)$, que es la profundidad máxima expresada como un porcentaje del diámetro de un circulo, cuyo área coincide con la del lago (Hutchinson, 1957). En el lago de Sanabria el valor de $Z_{\mathrm{r}}$ es $2.4 \%$, es un valor similar al de numerosos lagos (Tabla 2), indicando que los factores externos tienen una influencia relativamente alta sobre la estabilidad de la columna de agua.

\section{BIBLIOGRAFÍA}

ALDASORO, J. J., C. DE HOYOS \& J. C. VEGA, 1992. El lago de Sanabria. Estudio limnológico. Monografias de la red de espacios naturales de Castilla y León. J.M. Azcárate \& P. García (eds.).
Consejería de Medio Ambiente y Ordenación del Territorio (Junta de Castilla y León). Valladolid. $135 \mathrm{pp}$.

AMBROSETTI, W., L. BARBANTI, R. MOSELLO \& A. PUGNETTI, 1992. Limnological studies on the deep southern Alpine lakes Maggiore, Lugano, Como, Iseo and Garda. Mem. Ist. Ital. Idrobiol., 50: 117-146.

CATALÁN, J. 1987. Limnologia de l'estany Redó (Pirineu Central). Tesis Doctoral. Universidad de Barcelona. 230 pp.

DE HOYOS, C. 1996. Limnología del lago de Sanabria. Variabilidad interanual del fitoplancton. Tesis Doctoral. Universidad de Salamanca. $438 \mathrm{pp}$.

HAKANSON, L. 1981. A manual of Lake Morphometry. Springer-Verlag Ed. Berlin.

HUTCHINSON, G. E. 1957. A Treatise on Limnology. Vol 1. New York: John Wiley \& Sons. 1014 pp.

MARGALEF, R. 1983. Limnología. Ed. Omega. Barcelona. $1010 \mathrm{pp}$.

PLANAS, M. D. 1973. Composición, ciclo y productividad del fitoplancton del lago de Banyoles. Oecologia Aquatica, 1: 3-106

RICO, E., A. CHICOTE, M. E. GONZALEZ \& C. MONTES. 1995. Batimetría y análisis morfométrico del lago de Arreo (N. España). Limnetica, 11 (1): 55-58.

TABOADA, J. 1913. El lago de San Martín de Castañeda. Bol. Soc. Esp. Hist. Nat., 13: 860-883.

VEGA, J. C., C. DE HOYOS \& J. J. ALDASORO, 1992. The Sanabria lake. The largest natural freshwater lake in Spain. Limnetica, 8: 49-57

VEGA, J. C. \& J. J. ALDASORO, 1994. Geología de Sanabria. Monografias de la red de espacios naturales de Castilla y León. J.M. Azcárate \& $\mathrm{P}$. García (eds.).Consejería de Medio Ambiente y Ordenación del Territorio (Junta de Castilla y León). Valladolid. 47 pp. 
